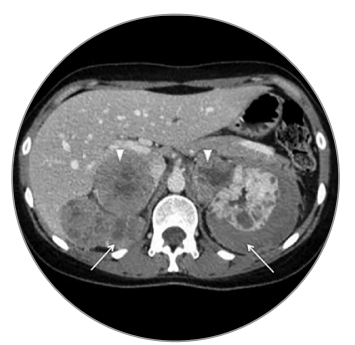

\section{Hipernefroma bilateral. Presentación de un caso}

\author{
Bilateral Hypernephroma. A Case Report
}

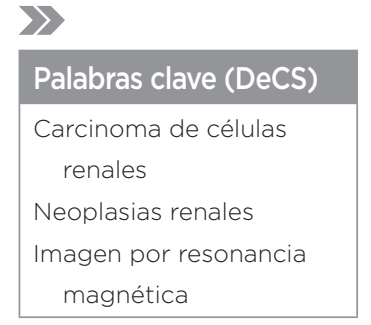

\section{Key words (MeSH)}

Carcinoma renal cell

Kidney neoplasms

Magnetic resonance

imaging
'Especialista en Radiología Intervencionista, Hospital Clínico Quirúrgico Docente "Hermanos Ameijeiras". La Habana, Cuba.

${ }^{2}$ Especialista en Radiología, Hospital Clínico Quirúrgico Docente: "Hermanos Ameijeiras", Universidad de Ciencias Médicas de La Habana, Cuba.

${ }^{3}$ Especialista en Neumología, Hospital Clínico Quirúrgico Docente "Hermanos Ameijeiras", Universidad de Ciencias Médicas de La Habana, Cuba.

${ }^{4}$ Especialista en Oncología, Hospital Clínico Quirúrgico Docente "Hermanos Ameijeiras", Universidad de Ciencias Médicas de La Habana, Cuba.

\title{
Resumen
}

El hipernefroma es un tumor renal común, más frecuente en hombres. Cuando es bilateral y hereditario tiene una conducta y manejo diferentes. En este caso se presenta una paciente femenina de 24 años diagnosticada hace un año con hipernefroma bilateral, mediante tomografía computarizada (TC) e imagen por resonancia magnética (RM), con componente hereditario y metástasis. Considerando los antecedentes familiares y con los resultados de los estudios radiológicos se realizó tratamiento sin complicaciones. La paciente se encuentra en seguimiento.

\section{Summary}

Hypernephroma is a common kidney cancer that has a higher frequency in men. When it is bilateral and hereditary it has a different behavior and treatment. In this case we present a 24 year old female patients who was diagnosed with bilateral hypernephroma one year ago, by means of computed tomography (CT) and magnetic resonance imaging (MRI) with hereditary components and metastasis. Considering the family's background and the use of radiological studies, the diagnosis and treatment was performed without complications. The patient is being followed up.

\section{Introducción}

El carcinoma de células renales (CCR) es el tumor renal más frecuente, con una incidencia de alrededor del $3 \%$ de todos los tumores malignos en adultos $(1,2)$. Dentro de ellos, la variedad histológica de células claras es el más frecuente (entre el 80-90 \%) (3). El tratamiento de elección es la cirugía, con exéresis del tumor o del riñón, aunque en los casos hereditarios, se debe prestar particular atención a tratar de maximizar la conservación de parénquima renal sin descuidar el riesgo de recurrencia. En el diagnóstico es esencial la tomografía axial computarizada (TAC), aunque puede ser útil realizar tomografía computarizada multidetector (TCMD) con técnica de angioTC para valorar vascularización del riñón cuando se plantea la cirugía conservadora de parénquima renal como opción (4). En una elevada proporción de casos tiene lugar unilateralmente; aproximadamente en el $5 \%$ ocurre en ambos riñones (5). El presente trabajo presenta y discute un caso diagnosticado como hipernefroma bilateral.

\section{Presentación de caso}

Paciente femenina de 24 años de edad, fumadora. Con antecedentes patológicos de hipotiroidismo diagnosticado hace un año y tratamiento con levotiroxina. Refiere padre fallecido por insuficiencia renal crónica e hipernefroma bilateral, a los 29 años de edad.
Paciente que inició relaciones sexuales a los 15 años, uso de anticonceptivos orales, acudió hace un año por amenorrea de dos meses de evolución. Los exámenes de laboratorio descartaron embarazo y diagnosticaron hipotiroidismo, continuó con amenorrea durante los últimos 12 meses. Hace tres meses acudió al servicio de oftalmología por disminución de la agudeza visual, marcada por el ojo izquierdo. En imagen por resonancia magnética (RM), se estableció diagnóstico presuntivo de macroadenomas de hipófisis, con el cual se intervino quirúrgicamente. El análisis histopatológico de la pieza quirúrgica fue negativo para adenoma hipofisario, que condujo a sospechar lesiones de etiología secundaria. Estuvo en terapia intensiva por 29 días en recuperación. Posteriormente acudió al hospital por dolor abdominal generalizado irradiado a la espalda.

Examen físico: Peso $70 \mathrm{~kg}$, talla $1,73 \mathrm{~cm}$, IMC de $23,3 \mathrm{~kg} / \mathrm{m}^{2}$.

Se le practicaron exámenes de laboratorio: hemoglobina $6 \mathrm{~g} / \mathrm{dL}$, por lo cual se transfundieron 2 unidades de glóbulos rojos empaquetados.

Se realizó angioTAC de tórax y abdomen que evidenció lesiones expansivas en ambos riñones, de predominio en lado derecho, con evidencia de hematomas perirrenales de moderada cuantía, asociado a lesiones heterogéneas en las glándulas suprarrenales de posible etiología secundaria y vasos de neoformación subcapsular o periférico, hallazgos indicativos de carcinoma de células renales de variedad células claras (figura 1). 

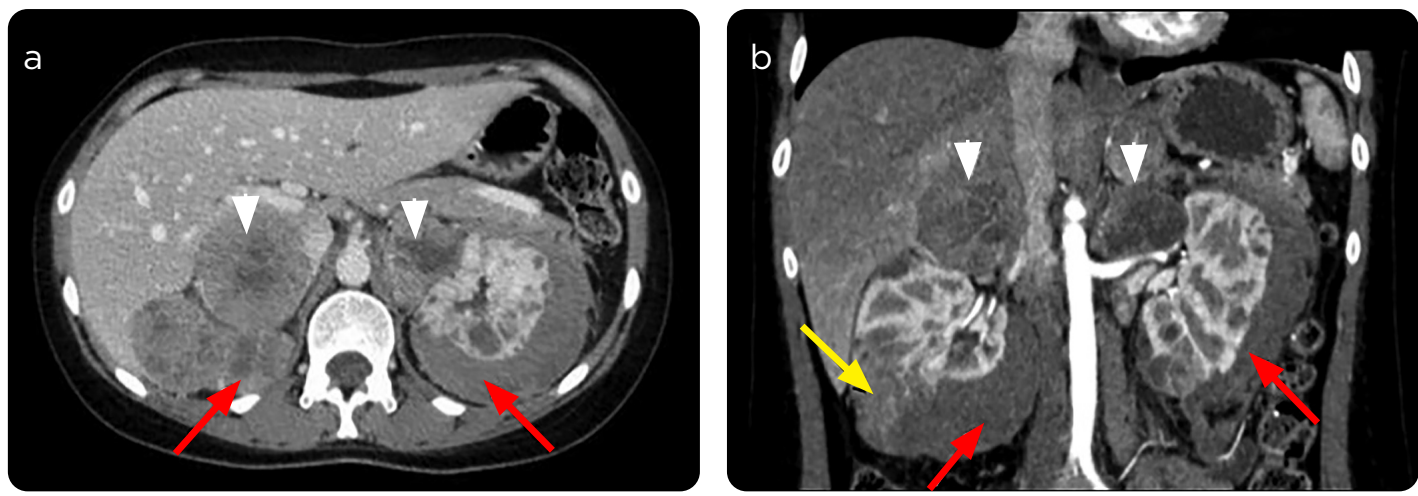

Figura 1.ay b) Angiotomografía de abdomen. Corte axial y reconstrucción coronal con proyección de máxima intensidad (MIP) Lesión expansiva tumoral vascularizada en polo inferior del riñón derecho (flecha amarilla). Hematoma subcapsular renal bilateral (flechas rojas). Ambas glándulas suprarrenales de aspecto metastásico (cabezas de flecha).
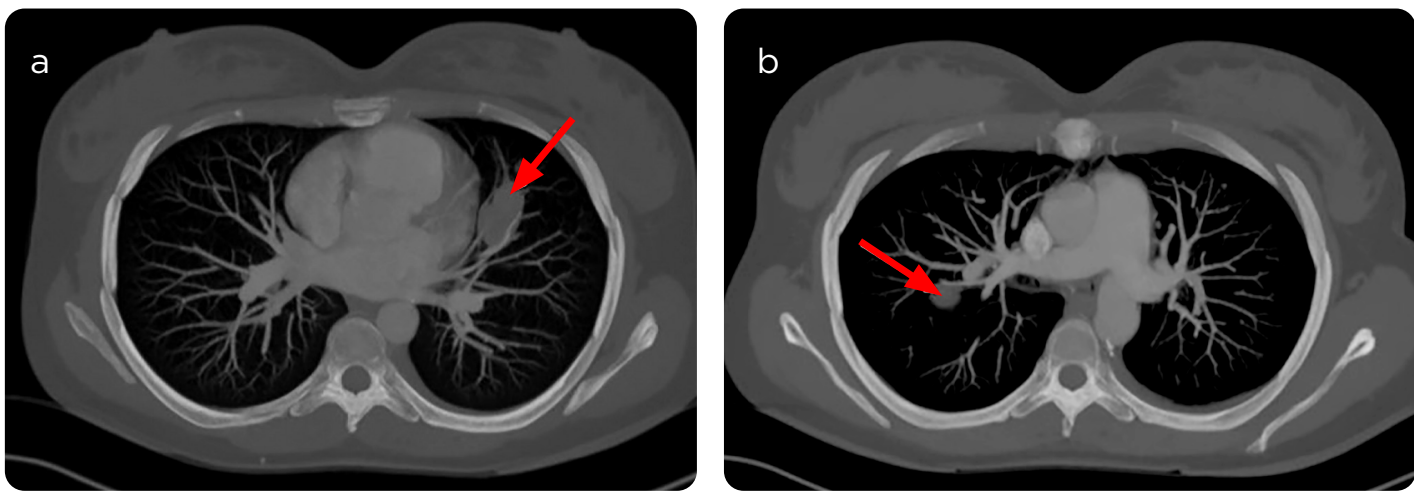

Figura 2. a y b) Angiotomografía de tórax. Cortes axiales con proyección de máxima intensidad (MIP). Imágenes nodulares de tipo metastásicas secundarias al CCR identificado (flechas rojas). Confirmado por biopsia pulmonar.
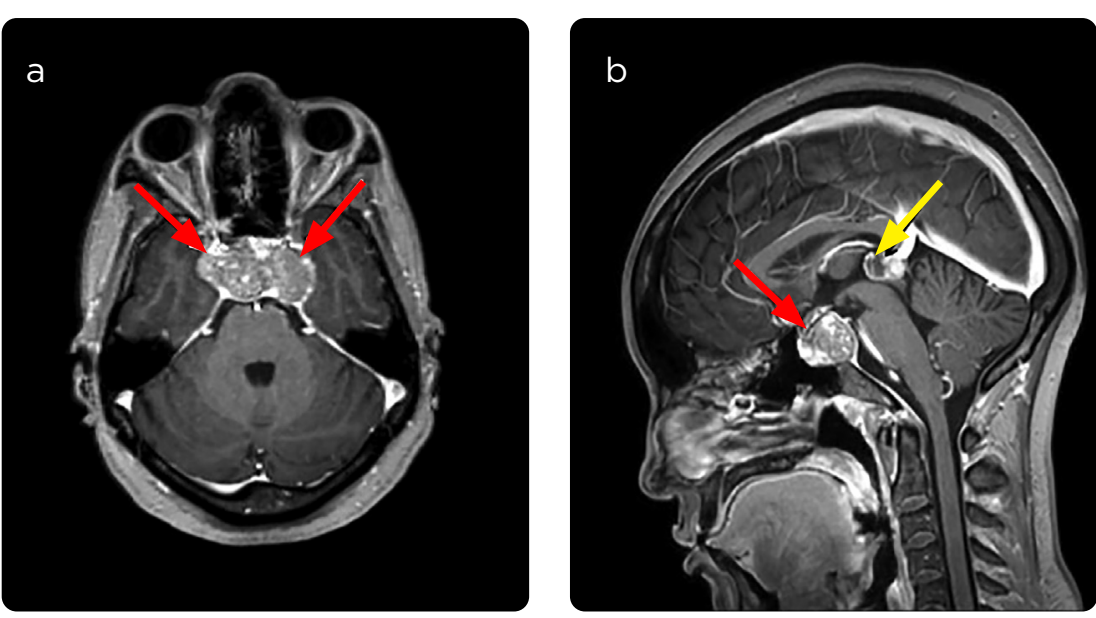

Figura 3. a y b) Imagen por resonancia magnética de cráneo, secuencias axial y sagital potenciadas en T1 con gadolinio. Tumoración vascularizada en región selar (flechas rojas) y en región pineal inferior al esplenio del cuerpo calloso (flecha amarilla).
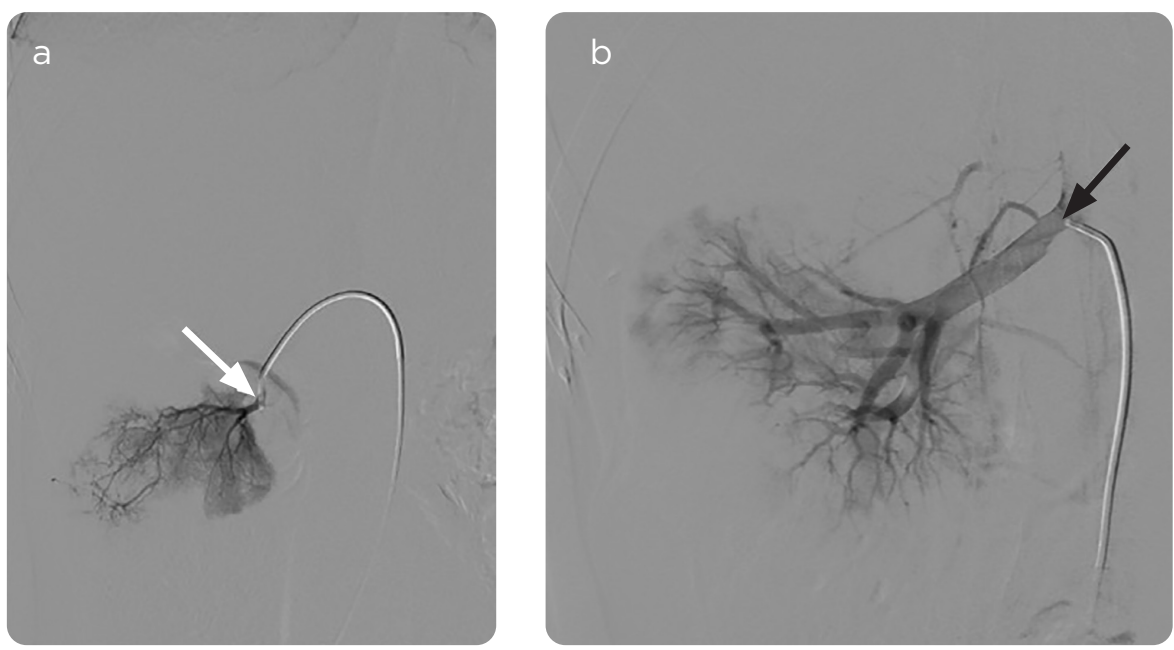

Figura 4. A) Cateterismo súper selectivo de rama aferente a polo inferior en plano frontal de riñón derecho (flecha blanca) donde se aprecia microvascularización distal con vasos tortuosos que forman fino entramado capilar, con múltiples intercomunicaciones, los cuales se proyectan en la porción más caudal y lateral del propio polo inferior del órgano, adoptando configuración nodular. b) Inyección proximal desde catéter angiográfico ubicado en el ostium de la arteria del riñón derecho (fase arterial tardía) (flecha negra) en donde es evidente la ausencia total de la neovascularización tumoral nodular antes observada, hacia la porción más caudal y lateral del polo inferior del órgano luego de la liberación de emboesferas de 300 micrones. 
Se evidenciaron, además, lesiones de aspecto nodular en ambos campos pulmonares del tórax (figura 2).

A los dos días se realizó imagen por RM de cerebro, secuencias con información T1, T2 y T1 con gadolinio, en las cuales se observó masa tumoral polilobulada en región selar, que destruye el dorso selar, así como las clinoides anteriores y posteriores con crecimiento supraselar y hacia la izquierda, donde comprime y desplaza al lóbulo temporal ipsilateral y al derecho. En su crecimiento anterior comprime el quiasma óptico, rompiendo el piso de la silla turca y con extensión al seno esfenoidal. En la región pineal se observa otra lesión de similares características (figura 3).

A la semana siguiente se realizó la embolización del hipernefroma del polo inferior del riñón derecho sin complicaciones (figura 4).

\section{Discusión}

La incidencia de hipernefroma bilateral sincrónico es de aproximadamente el $2 \%$ de los individuos con CCR oscilando entre el $1 \%-4 \%$ (6). El CCR bilateral es más común en pacientes con antecedentes familiares de CCR, en los cuales se ha identificado una mutación genética específica (7).

El hipernefroma se informa con mayor frecuencia en hombres a razón de 3:1 y más comúnmente entre los 50-70 años de edad. Es de causa desconocida, aunque se han identificado algunos factores de riesgo, como el tabaquismo y la historia familiar (4). El caso presentado es una mujer de 24 años de edad, que no está en el rango de lo que se considera más común. Sin embargo, se corresponde con la edad en la que ocurren los hipernefromas hereditarios, que son el mayor grupo cuando el tumor es bilateral.

Los pacientes con CCR permanecen generalmente asintomáticos, hasta estadios avanzados de la enfermedad. Entre el $25-30 \%$ se diagnostican por síntomas asociados a las metástasis (4). Hay gran diversidad de manifestaciones clínicas entre las que se encuentran la forma dolorosa lumbar y abdominal y la forma anémica, ambas manifestaciones presentes en este caso.

Además de la angiografía convencional, la TAC con reconstrucción tridimensional y la TCMD con técnica de angioTC permiten la evaluación vascular detallada y eventual programación de tratamiento conservador o embolización. El caso presentado se diagnosticó usando angioTC e IRM, y se confirmó masa tumoral en ambos riñones, además de lesiones en glándulas suprarrenales, lesiones nodulares en ambos campos pulmonares y lesiones cerebrales de aspecto secundario.

En los pacientes con tumores renales unilaterales, el objetivo de la terapia es la cura, con maximización de la función renal, para lo cual la cirugía puede ser suficiente. En los casos bilaterales hereditarios, el objetivo de la terapia es prevenir la diseminación del cáncer preservando en lo posible la función renal y limitando el número de cirugías (6). Se sugiere considerar la nefrectomía parcial, si potencialmente disminuye el riesgo de diálisis. En el caso presentado, se decidió nefrectomía parcial en el lado de mayor complejidad y tamaño.

En una serie de 128 casos (8) con cirugía bilateral, el $68 \%$ tuvo necesidad de una nueva cirugía renal. La supervivencia global fue del $88 \%$ a los 16 años y la supervivencia de CCR específica del $97 \%$. Este trabajo sugiere un tiempo mínimo de seguimiento de 10 años luego de la cirugía inicial.

\section{Conclusiones}

El hipernefroma bilateral es relativamente raro y de difícil manejo, debido a los agravantes de morbilidad y mortalidad cardiovascular asociados y al riesgo de diálisis, tanto como a la malignidad renal. Resultan de vital importancia las técnicas radiológicas en el diagnóstico y previo a la cirugía, con el objetivo de prevenir las complicaciones mediante el estudio de la anatomía vascular.

En el caso presentado, los exámenes radiológicos concluyen el diagnóstico y se adopta conducta de nefrectomía parcial.

\section{Referencias}

1. Siegel RL, Miller KD, Jemal A. Cancer statistics. CA Cancer J Clin. 2015;65:5-29.

2. Pérez García F, Regadera Sejas F, Rodríguez Martínez J, Martínez Gómez F. Adenocarcinoma renal bilateral sincrónico: Nuestra experiencia con la cirugía conservadora renal. Arch Esp Urol. 1999;5(3):471-7.

3. Cheng W, Farow G, Zincke H. The incidence of multicentricity in renal carcinoma. J Urol. 1997;146(6):1221-9.

4. Cameron Nelson GA, Ríos Sanabria C. Hipernefroma. Revista Médica de Costa Rica y Centroamérica. 2015:62(615):281-6.

5. Wiklund F, Tretli S, Choueiri TK, Signoretti S, Fall K, Adami HO. Risk of bilateral renal cell cancer. J Clin Oncol. 2009;27:3737-41.

6. Shuch B, Singer EA, Bratslavsky G. The surgical approach to multifocal renal cancers: hereditary syndromes, ipsilateral multifocality, and bilateral tumors. Urol Clin North Am. 2012;39:133-48.

7. Qi N, Li T, Ning X, Peng X, Cai L, Gong K. Clinicopathological features and prognosis of sporadic bilateral renal cell carcinoma: a series of 148 cases. Clin Genitourinary Cancer. 2017. doi: 10.1016/j.clgc.2017.03.008.

8. Singer EA, Vourganti S, Lin K, et al. Outcomes of patients with surgically trated bilateral renal masses and a minimum of 10 years of follow-up: the NCI experience. J Urol. 2011;185(Suppl 4):e746.

\section{Correspondencia}

\author{
José Luis Sánchez Peña
}

Universidad de Ciencias Médicas de La Habana (Cuba)

Calle 146 \# 2504, entre 25 y 31

Siboney, Playa, La Habana, 11300, Cuba

mdjsp@hotmail.com

Recibido para evaluación: 10 de enero de 2020

Aceptado para publicación: 10 de febrero de 2020 